\title{
Using Balloons to Model Pi-Conjugated Systems and to Teach Frontier Molecular Orbital Theory
}

\author{
Daniel J. Swartling* , Janet G. Coonce, Derek J. Cashman \\ Department of Chemistry, Tennessee Tech University, 55 University Drive, Cookeville, TN 38505 United States \\ *Corresponding author: dswart@tntech.edu
}

\begin{abstract}
Large-scale molecular orbital balloon models have been designed and developed for implementation in the general, organic, or physical chemistry classroom. The purposes of the models are to help students visualize and understand concepts of pi-bonding, conjugation, aromaticity, and cycloaddition reactions or symmetry-controlled reactions. Second-semester organic chemistry students have welcomed the models with positive responses, claiming that the 3D models bring 2D textbook and lecture images to life. The balloon models may be constructed and presented by the instructor during a formal lecture, or they may be constructed by students during problem-solving workshops. Short video tutorials have been created to demonstrate the construction of these inexpensive classroom manipulatives.
\end{abstract}

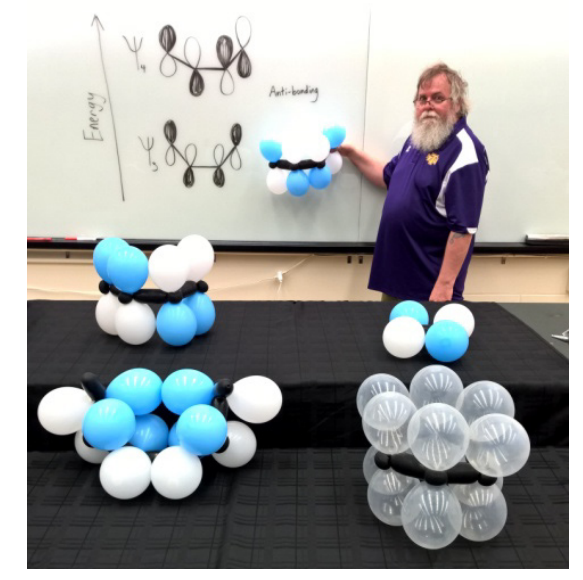

Keywords: High School / Introductory Chemistry, First-Year Undergraduate / General, Second-Year Undergraduate, Upper-Division Undergraduate, Demonstration, Organic Chemistry, Collaborative/Cooperative learning, Computer-Based Learning, Hands-On Learning/Manipulatives, Alkenes, Alkynes, Aromatic Compounds, MO Theory

Cite This Article: Daniel J. Swartling, Janet G. Coonce, and Derek J. Cashman, "Using Balloons to Model Pi-Conjugated Systems and to Teach Frontier Molecular Orbital Theory." World Journal of Chemical Education, vol. 6, no. 2 (2018): 102-106. doi: 10.12691/wjce-6-2-5.

\section{Introduction}

Why Balloons? Our student affiliate chapter of the American Chemical Society [1] successfully uses balloons for public outreach and fundraising activities. Balloons do not require much storage room, but when they are inflated, they are large enough to see from a great distance. Children and adults alike are intrigued by balloons, and balloons have a strong connection to chemistry: balloons are made from a polymer (polyisoprene), which is a natural product (latex) and is "green" (biodegradable). There have been many papers published regarding the use of balloons as an aid in teaching various chemical concepts. Examples include: Charles' Law, [2,3] Avogadro's Law, [4] molar mass, [5] and the ever-popular hydrogen balloon explosion. [6] Balloons have also been used teach VSEPR theory, [7] atomic orbitals, [8] and the modeling of organic molecules. [9] Balloons of different shapes and sizes are readily available at party stores, large retail stores, and online venders [10].

\section{Orbital Models}

Line-angle formulas rapidly communicate ideas and molecular structures, but they are often lacking when used to explain orbital interactions. By using 260 modeling "twist" balloons and 5-inch round balloons, [11] tangible models can be quickly constructed in class to help students visualize the concepts of pi-bonding, conjugated systems, and molecular orbital (MO) theory (Figure 1). 


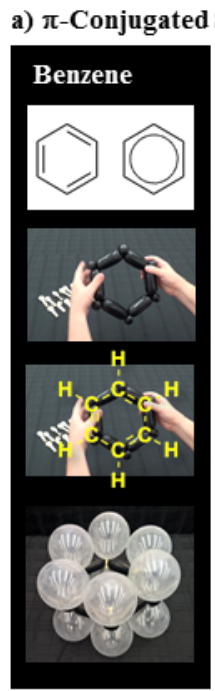

b) Frontier Molecular Orbital Theory
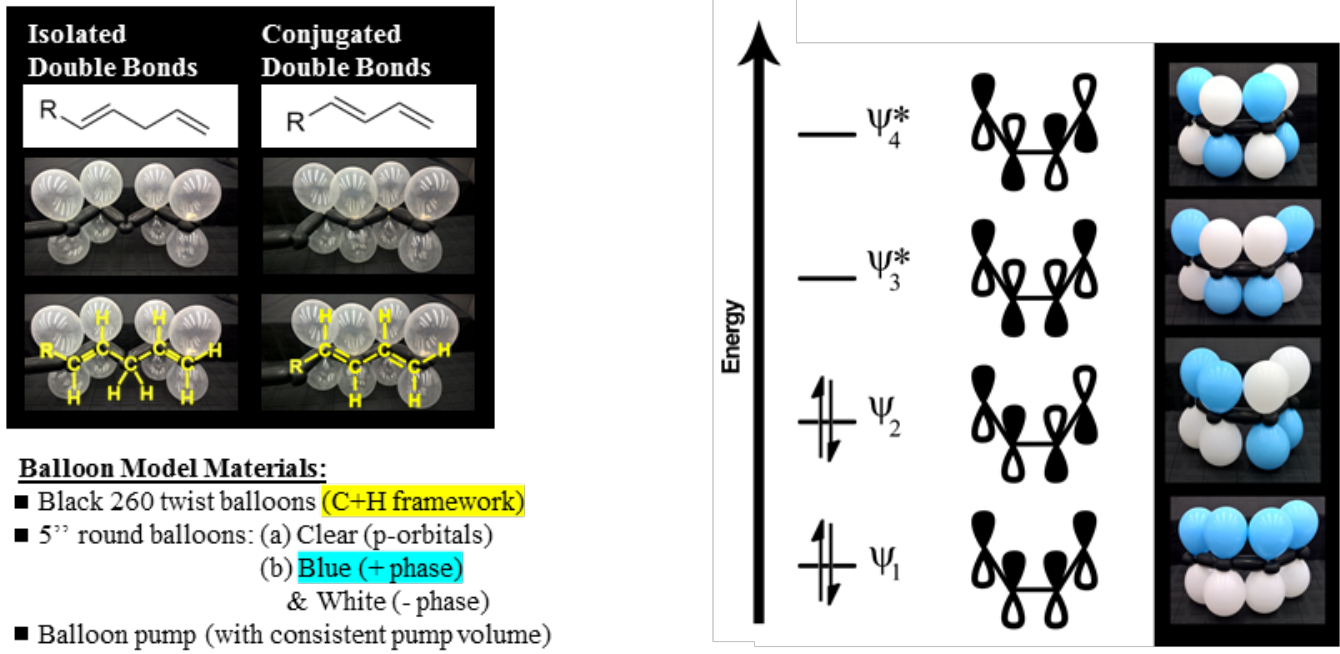

Figure 1. Balloon models to describe (a) pi-conjugated systems and (b) frontier molecular orbital theory

\subsection{Pi-Bonding}

To model a p-orbital, simply tie two equally-inflated clear 5' balloons together. The knot region represents the atom's nucleus, and the balloon interiors represent the regions of space with a probability of finding an electron. Place two p-orbitals next to one another to model a pibond, emphasizing the electron orbital overlap both above and below the atomic nuclei. Then, use these hands-on balloon manipulatives to help students visualize the pi bond of an alkene's carbon double bond and the two pi bonds of an alkyne's carbon-carbon triple bond (Figure 2).
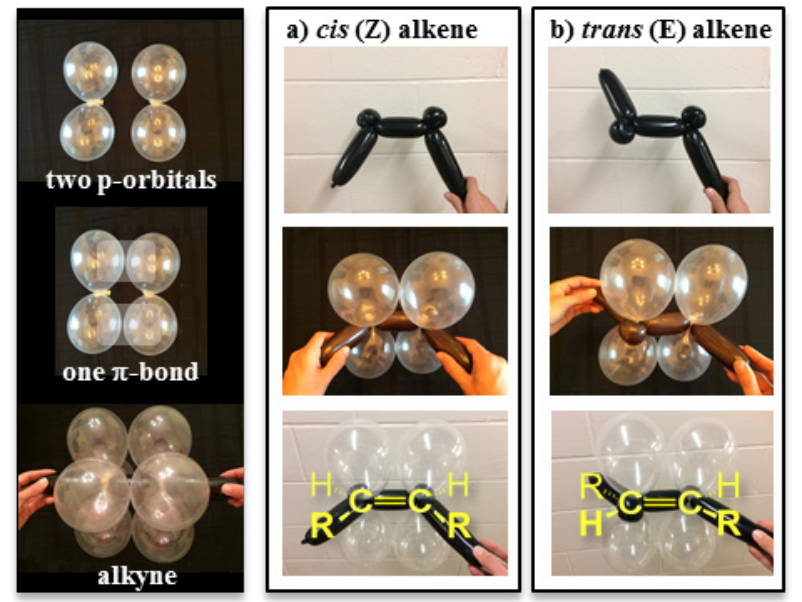

Figure 2. Clear balloon models to represent a single $\pi$-bond with electron overlap above and below the atomic nuclei.

While there may be a learning curve associated with balloon-twisting, one of the co-authors of this publication was able to successfully construct all of the models presented here within a 24-hour time period with no issues and no prior experience in balloon-twisting by studying the videos presented in (4. Associated Content).

\subsection{Cis and Trans Alkenes}

To model cis (Z) and trans (E) alkenes, start by making a twist approximately 4" from the knot of a partially inflated black 260 "twist" balloon. Make a second twist 5" from the knot to create a 1" bubble. Squeeze these two twists into a pinch twist to model a carbon atom. Study the videos (see 4. Associated Content) for demonstrations of the complete construction of these models. The alkene models were designed to explain the lack of free rotation around the carbon-carbon double bond. A cis alkene model can quickly be repositioned into a trans alkene, but the pi bond must be broken in the process. (Figure $2 \mathrm{a} \& \mathrm{~b}$ ).

\subsection{Pi-Conjugated Systems}

Benzene is often problematic to visualize, since we draw it in either the Kekule form or as the modern hybrid structures (Figure 1a). Students often envision that the electrons are racing around inside the 6-membered ring, oblivious to the orbitals involved, and have a hard time visualizing an overlapping cloud of electrons above and below the ring. This $3 \mathrm{D}$ structure is clearly demonstrated by making a balloon model of benzene using a black 260 "twist" balloon for the 6-membered ring and 12 clear 5 -inch round balloons for the p-orbitals and overlapping pi-system (Figure 1a).

\subsection{Isolated vs. Conjugated Double Bonds}

Next, consider the structures for 1,4-pentadiene and 1,3 pentadiene. The former has two isolated double bonds, while the latter has two conjugated double bonds. Many students forget that a double bond is comprised of a sigma bond and a pi bond, and it is the overlap of the pi system as a whole that accounts for conjugation. By modeling the systems out of black 260 "twist" balloons for the carbon backbone and using 5-inch clear round balloons for the p orbitals, this is more readily apparent (Figure 1a).

So far we have seen that we can model benzene, dienes, and conjugated dienes very quickly and easily using balloons. These can be built prior to lecture, or for more impact, during lecture. Since many students learn best by a hands-on approach, allow the students to build the models themselves. If everyone in the group assists in blowing up the balloons, the models can be put together in only a few minutes. When students are challenged to use the balloons to solve problems, the models can be used 
as a productive student workshop. Colored balloons can be used to model the positive and negative phases of molecular orbitals.

\subsection{Frontier-Molecular Orbital Theory}

A molecular orbital (MO) is a mathematical function that describes the wave-like behavior of an electron in a molecule. This function is used to calculate the probability of finding an electron in any specific region. As such, an orbital is not a solid structure or particle. Therefore, constructing a model of an orbital using a gas-filled balloon, with particles (in a gas, atoms or molecules) moving about at high speed would be more representative of what an orbital actually represents than a 3D-printed, solid model of the wave function calculated using quantum chemistry software. Furthermore, balloon models allow students to build and visualize the orbitals involved in symmetry-controlled reactions. Using Frontier Molecular Orbital Theory and the Woodward-Hoffman rules, students can tell if a given reaction will take place and, if allowed, predict the stereochemical outcome. The classic textbook example often used is looking at the molecular orbitals of butadiene. For example, Sykes', "A Guidebook to Mechanisms in Organic Chemistry," contains a 2D diagram of the four-MOs arising from the four p-AOs in butadiene in the cisoid conformation (Figure 1b, black and white drawing). [12]

There are a number of excellent quantum chemistry (QM) software packages currently available that are capable of calculating and visualizing molecular orbitals. This software ranges from the relatively easy-to-use complete commercial software package, Hyperchem (Hypercube, Inc.; Gainesville, FL, USA) to freely available software such as Avogadro, [13] which must be used in conjunction with a separately-obtained quantum chemistry program such as Gaussian (Gaussian, Inc.; Wallingford, CT, USA) or GAMESS (Ames Laboratory, Iowa State University; Ames, IA, USA) to perform the necessary Quantum Mechanical (QM) calculations. While GAMESS is free for academic users, Gaussian is commercial software and requires the purchase of a software license. MOLDEN is another free software package that may be utilized for the visualization of QM calculations from both Gaussian and GAMESS. [14] The advantage of using these software packages for molecular orbital calculations is their speed and accuracy. The disadvantage is that there may be a cost which could limit its use in the classroom, and while free options do exist, the necessary configuration of the software to perform the molecular orbital calculations required are not trivial and require extra computational chemistry or coding knowledge that would limit their applicability in undergraduate level chemistry courses.

Furthermore, computational chemistry software packages are limited in calculating and visualizing atomic and molecular orbitals on a single molecule. It is more complicated and may require multiple and specialized visualization software to display the molecular orbitals of two or more molecules interacting with each other in, for example, a Diels-Alder reaction. Additionally, such a presentation would nonetheless be a two-dimensional representation on a projector screen of a three-dimensional concept. While technology does exist for visualizing actual 3D concepts in virtual reality environments, this is not always practical in an undergraduate classroom environment. The same set of MOs can be built using black 260 "twist" balloons for the carbon framework and two different colored 5-inch round balloons for the orbitals, one color representing $(+)$ and the other color representing (-). The balloon models are shown in Figure 3.

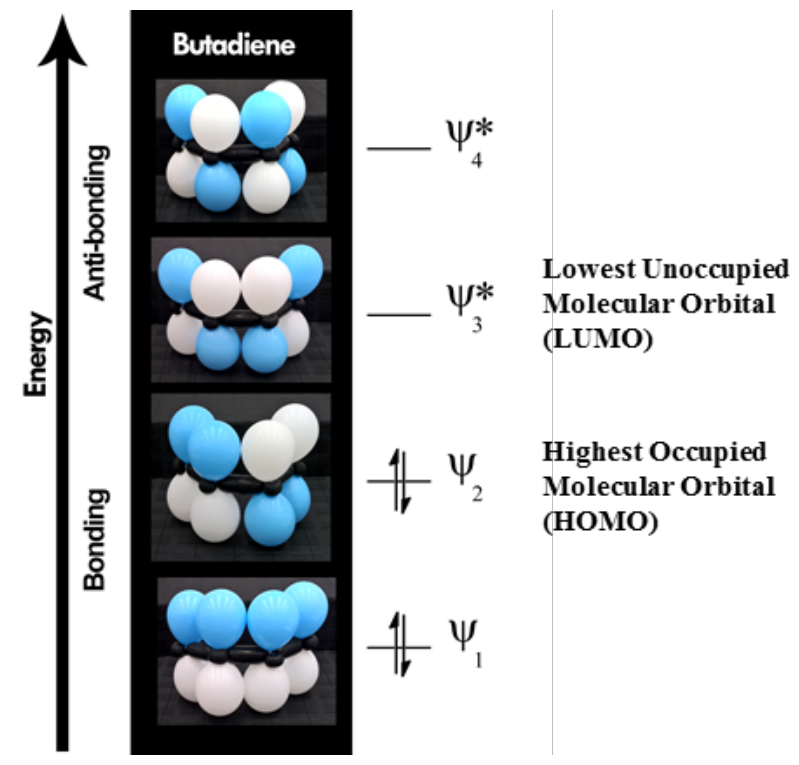

Figure 3. Using balloon models to identify the bonding and anti-bonding MOs of butadiene

A main concept for students to realize when looking at the phase of orbitals is that only orbitals of the same phase will overlap and lead to bond formation. The models can be used to look at reactions using the frontier orbital approach, where the Highest Occupied Molecular Orbital (HOMO) of one reactant is seen as overlapping with the Lowest Unoccupied Molecular Orbital (LUMO) of the other reactant. If a reaction is unimolecular, only the HOMO has to be considered. Through the use of balloons, students can quickly build HOMO and LUMO models of orientation and then predict the outcome of a given reaction. Only one set of MOs needs to be built for each reactant; the 5-inch round balloons can be quickly repositioned to create the proper phase.

\subsection{Cycloaddition Reactions}

The Diels-Alder reaction is a well-known [4+2] cycloaddition reaction. In its simplest form it can be illustrated by involving the HOMO of 1,3-butadiene and the LUMO of ethylene. This can be visualized by the two balloon models shown in Figure 4.

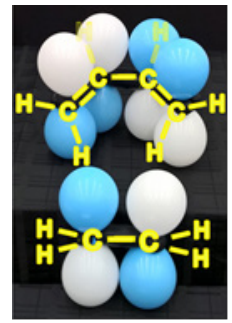

HOMO of Butadiene

LUMO of Ethylene

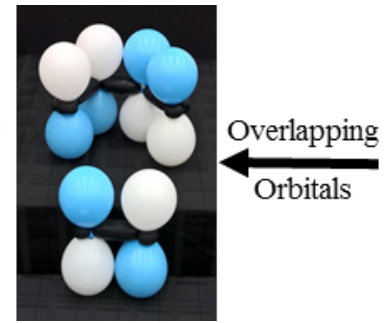

Figure 4. HOMO-LUMO interaction in the Diels-Alder reaction 


\subsection{Electrocyclic Reactions}

A reaction often used in textbooks to illustrate electrocyclic reactions is the cyclisation of trans, cis, trans-2,4,6-octatriene, which has different outcomes if done thermally or photochemically. [15] The question posed to students is: what is the product of the thermal electrocyclic reaction, the cis- or the trans-dimethyl cyclohexadiene, shown in Figure 5.
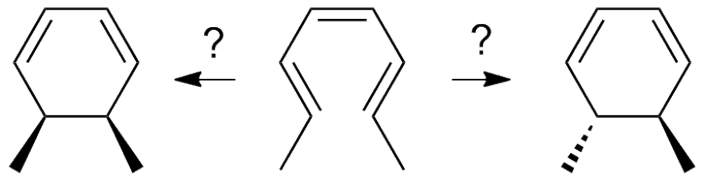

Figure 5. Which is the correct product for the thermal reaction?

First, students must build a balloon model of the HOMO, as shown in Figure 6.

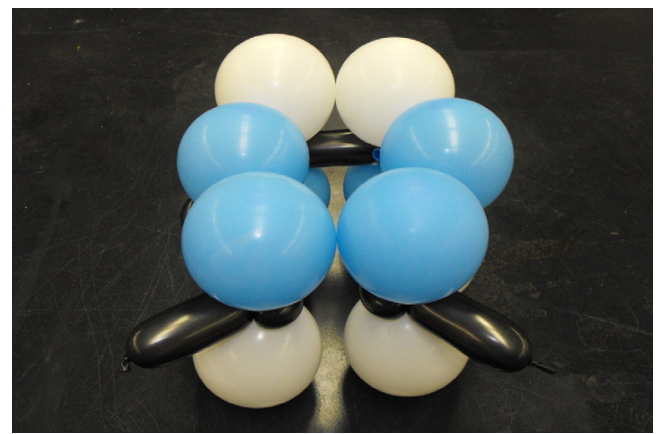

Figure 6. The HOMO for $(2 E, 4 Z, 6 E)-2,4,6$-octatriene.

According to the Woodward-Hoffman rules, for a $4 n+2$ system under thermal conditions, the orbital motion for bonding is dis-rotatory. Rotating the two outermost $p$ orbital balloons so that lobes of the same color overlap is a dis-rotatory motion, and shows the methyl groups ending up cis, as shown in Figure 7.

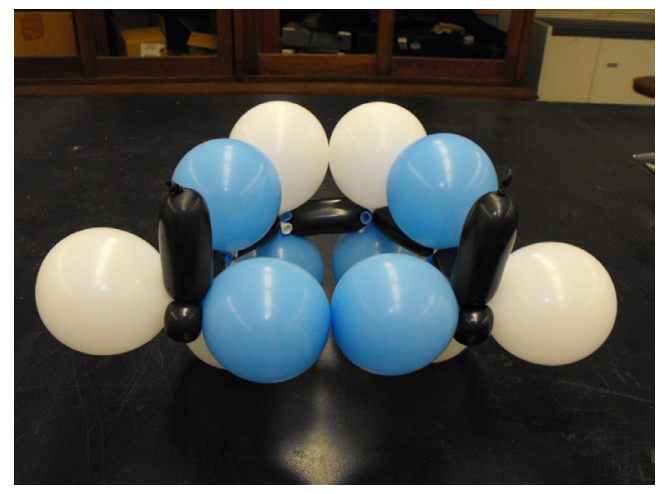

Figure 7. The disrotatory motion of the like phases results in a bonding situation, and shows both methyl groups pointing up above the newlyformed ring

\subsection{Student Responses}

Students enrolled in a second-semester organic chemistry class were given a textbook assignment to read about pi-conjugated systems with an introduction to molecular orbital theory as it relates to the Diels-Alder reaction. After this short introduction, an optional pre-lecture quiz was given to the class. Institutional
Review Board guidelines for research with human subjects were followed in this study, enabling us to report feedback from this study.

The student average of the pre-quiz was $24.6 \% \pm 1.9 \%$ $(n=26)$ Then, the balloon models described in this paper were presented to the students as objects for classroom discussion. After experiencing and manipulating the balloon models in a lecture environment, the students re-took the optional quiz. The student average $(n=26)$ on the post-activity quiz was $86.9 \% \pm 1.6 \%$.

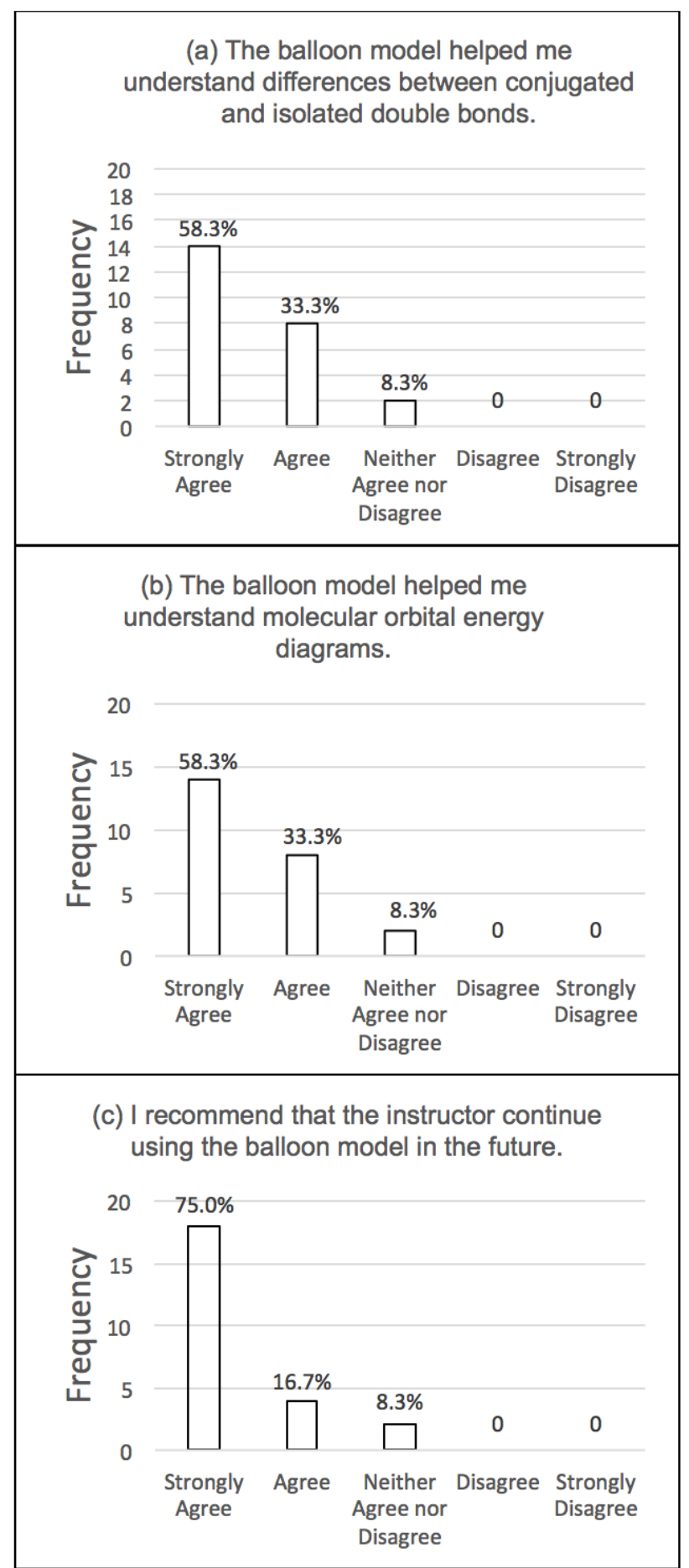

Figure 8. Student survey responses after experiencing the balloon models in an organic chemistry classroom

Students commented that the balloon models were, "helpful for visual learners like myself," and "the balloons 
honestly helped." Of the 24 students who agreed to take the survey, $22(92 \%)$ either agreed or strongly agreed that (a) "The balloon model helped me understand the differences between conjugated and isolated double bonds.", (b) "The balloon model helped me understand molecular orbital energy diagrams," and (c) "I recommend that the instructor continue using the balloon model in the future." (Figure 8).

\section{Conclusions}

It can be difficult to draw orbital interactions on the chalkboard in such a way as to have all students grasp what is taking place. Using balloons to illustrate orbital concepts is very quick and simple. Allowing the students to build HOMOs and LUMOs themselves and guide themselves, or group study sessions, additionally helps to develop self-confidence and critical thinking skills. Balloons and a pump are relatively inexpensive, do not require a computer or expensive software or require advanced computational knowledge and expertise, and can be used by the students wherever they congregate to study. The balloon models make learning the material a fun and engaging activity for students of general, organic, and/or physical chemistry. Balloons will occasionally pop while being inflated, but that only adds to the fun!

\section{Associated Content}

Instructional Video Tutorial Links to demonstrate how to make the balloon orbital models described in the paper. Filmed, edited, and produced by Janet Coonce and Dan Swartling for publication in this paper.

\section{A. Benzene}

https://youtu.be/icaE3JSUVhM

B. Conjugated \& Isolated Double Bonds:

https://youtu.be/pqSUEH-V8Ao

C. Highest Occupied Molecular Orbital (HOMO) of Butadiene

https://youtu.be/2cJ9xjrk3IM

D. Lowest Unoccupied Molecular Orbital (LUMO) of Ethylene

https://youtu.be/pVU-f8cuTOo

\section{Acknowledgments}

The authors wish to thank Tennessee Technological University for the lecture hall access for video recording and balloon activities. We also want to thank the student participants that agreed to provide feedback for the use of the balloons in a second-semester organic chemistry classroom.

\section{References}

[1] Student Members of the American Chemical Society - Tennessee Tech Chapter.

[2] Carney, G.D.; Kern, C.W.; Balloon Balance Thermometer: A Lecture Demonstration of Charles' Law. J. Chem. Educ., 1979, 56 823-824.

[3] Deese, William C. A Miniature Hot Air Balloon and Charles' Law J. Chem. Educ., 1990, 67, 672 .

[4] Fenster, Ariel A.; Harpp, David N.; Schwarcz, Joseph A. A Useful Balloon Demonstration: Pressure Difference Behavior. J. Chem. Educ., 1986, 63, 629.

[5] Jackson, Barbara Albers; Crouse, David J. Experimentally Determining the Molar Mass of Carbon Dioxide Using a Mylar Balloon. J. Chem. Educ., 1998, 75, 997-998.

[6] Lawrence, Stephen S. Modified Hydrogen Balloon Explosion. J. Chem. Educ., 1995, 72, 177.

[7] Roberts, Royston M.; Traynham, James G. Molecular Geometry: As Easy As Blowing Up Balloons. J. Chem. Educ., 1976, 53, 233-234.

[8] Hoogenboom, Bernard E. Three-Dimensional Models of Atomic Orbitals. J. Chem. Educ., 1962, 39, 40-41.

[9] Niac, G. Balloon Models for Organic Molecules. J. Chem. Educ., $1978,53,303$

[10] Two online sources for balloons are: T. Myers Magic, Inc. 6513 Thomas Springs Rd., Austin, TX 78736, http://www.tmyers.com/; and La Rock's Fun and Magic Outlet, 1511 S. Industrial Park Rd., Lincolnton, NC 28092, http://www.larocks.com/.

[11] A " 260 " balloon model is a balloon that, when fully inflated, is 60 inches long and approximately 2 inches in diameter.

[12] Sykes, Peter. A Guidebook to Mechanisms in Organic Chemistry, Sixth Edition, (c)1986 Longman Group, Ltd., Essex, England. ISBN 0-582-44695-3. Page 344.

[13] Hanwell, M.D.; Curtis, D.E.; Lonie, D.C.; Vandermeersch, T.; Zurek, E.; Hutchison, G.P. Avogadro: An advanced semantic chemical editor, visualization, and analysis platform. J. Chem. Inf., 2012, 4, 1-17.

[14] Schaftenaar, G.; Noordik, J.H.; Molden: A pre- and post-processing program for molecular and electronic structures. J. ComputerAided Molec. Des., 2000, 14, 123-134.

[15] Sykes, Peter. A Guidebook to Mechanisms in Organic Chemistry, Sixth Edition, (C)1986 Longman Group, Ltd., Essex, England. ISBN 0-582-44695-3. Page 345. 